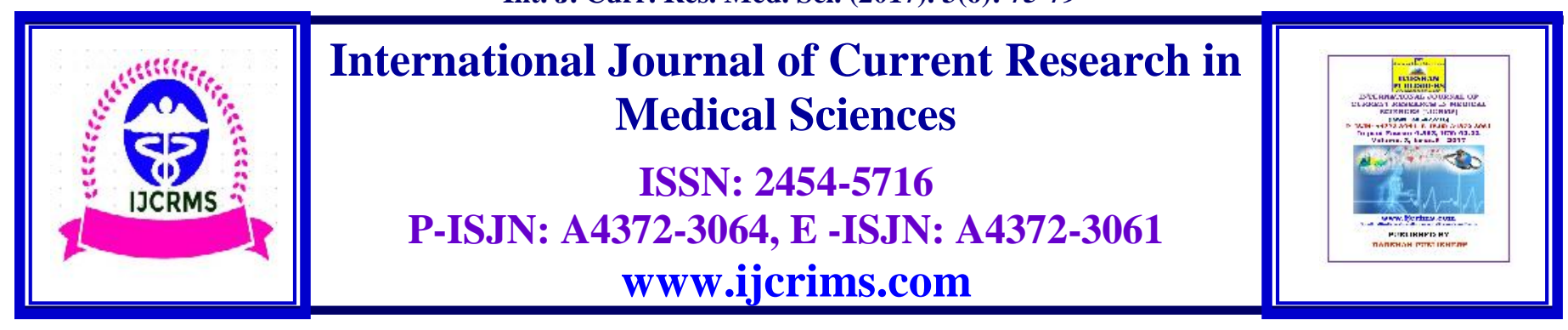

Case Report

Volume 3, Issue 6 -2017

DOI: http://dx.doi.org/10.22192/ijcrms.2017.03.06.010

\title{
A Case report of Oral lipoma on gingiva - A rare histological entity
}

\author{
* Chhaya Mangla, **Sukhpal Kaur, ${ }^{* * *}$ Riponjot Singh, \\ *****Harpreet Kaur Dhanju \\ *Periodontist, Shri Balaji Dental Clinic, Rohtak, Haryana. 124001. (Ex Postgraduate Student, \\ Dept.of Periodontology, Govt. Dental College, Patiala, Punjab, India) \\ ** Reader, Dept of orthodontics \& Dentofacial Orthopaedics, Desh Bhagat Dental College and Hospital, \\ Muktsar, Punjab, India, 152026 \\ *** Second year Dental Hygiene Student, Georgian College of Applied Arts and Technology, Barrie, Canada \\ ****Chief Dental Officer, Bhai Ghanayia Hospital, Amritsar, Punjab, India, 143001 \\ Corresponding Author: Sukhpal Kaur, Reader, Dept. of Orthodontics and Dentofacial Orthopaedics, \\ Desh Bhagat Dental College and Hospital, Muktsar, Punjab, India, 152026. \\ E-mail:docs284@gmail.com

\begin{abstract}
Lipomas are adipose mesenchymal neoplasms that rarely occur within oral cavity (1\% to $4 \%$ ). Oral lipomas are usually slow growing lesions with asymptomatic clinical course until they get larger in size. Although benign in nature, their progressive growth may cause interference with speech, mastication and aesthetics. Thus, their knowledge and prompt treatment is very important. The majority of oral lipomas are found on the buccal mucosa followed by tongue. We here present a case of oral lipoma on the gingiva in a 65yr old female.
\end{abstract}

Keywords: Lipoma, tongue, mesenchymal neoplasm, mastication.

\section{Introduction}

Lipomas are common soft tissue mesenchymal neoplasms, with 15-20\% of cases involving the head and neck region and only $1-4 \%$ affecting the oral cavity. The pathogenesis of lipoma is uncertain, but they appear to be more common in obese people. However, the metabolism of lipoma is completely independent of the normal body fat.
If the caloric intake is reduced, lipomas do not decrease in size, although normal body fat may be lost. Lipomas are slowly enlarging, with a soft, smooth-surface mass of the submucosal tissues. When it is superficial, there is a yellow surface discoloration. The lesion may be pedunculated or sessile and occasional cases show surface 
bosselation. Although its etiology is unknown, possible causes may include trauma, infection, chronic irritation and hormone alterations. In few cases of lipoma, rearrangement of $12 q, 13 q, 6 p$ chromosomes have been observed. ${ }^{1}$ There are several microscopic variants of lipoma. According to their histopathologic aspects, these can be characterized as, classic lipoma, fibrolipoma, intramuscular lipoma, angiolipoma, myxoid lipomas, spindle-cell lipoma, pleomorphic lipoma. ${ }^{2,5}$ Definitive diagnosis depends on correlation between the histological and clinical features. The histopathology remains the gold standard in the diagnosis of lipoma. ${ }^{1}$ The complete resection should be emphasized, which is the key factor to avoid reoccurrence. The first description of an oral lesion was provided in 1848 by Roux in a review of alveolar masses, where he referred it as a" yellow epulis" It is usually found in adults. The female to male ratio for all lipomas are 2:1 but oral lipomas occur more in men than women. The most common site of oral lipomas is the oral mucosa, a region rich in fatty tissue, followed by the tongue, lips, floor of the mouth, palate and gingival. This pattern corresponds to the quantity of fat deposits in the oral cavity. ${ }^{4} \mathrm{~A}$ case of oral lipoma on gingival is reported here for its rarity.

\section{Case history}

A $65 \mathrm{yr}$ female reported to the Department of Periodontology, with a chief complaint of painless swelling on the lingual gingiva of mandibular left third molar area for the last 10 months which had been gradually increasing (Figure 1). Though, she had no difficulty in mastication, speech and deglutition but she was aware of the swelling and anxious about the nature of the growth. The swelling was not associated with pain or bleeding. There was no associated fever, weight loss or other otolaryngeal problems.

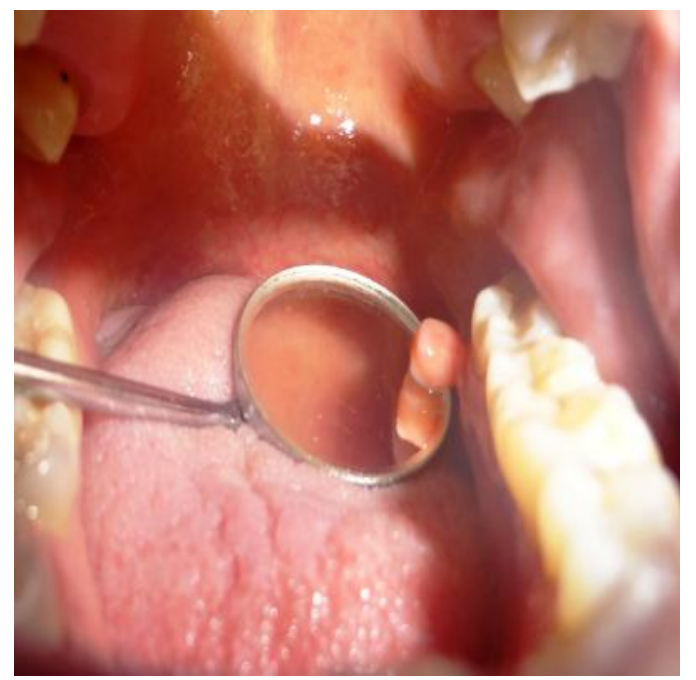

Figure 1: Showing lipoma on the lingual gingiva of mandibular left third molar.

On intraoral examination, a well defined round growth about $3-4 \mathrm{~mm}$ away from the marginal gingiva on the lingual side of the mandibular left third molar area and was about $1.5 \times 2 \mathrm{~cm}$ in size was seen. The growth was pedunculated, pinkish in color with yellowish tinge. On palpation the growth was rubbery soft, fluctuant, non tender, mobile, the margins were slippery under the palpating finger.
Caries and pain on percussion were not seen with respect to that tooth. Pulp vitality tests indicated that tooth was vital. Intraoral periapical radiograph with respect to mandibular left third molar showed that there was no evidence of crestal bone loss and lamina dura was intact around the roots of $3^{\text {rd }}$ molar. The patient was normally built for her age with no defect in gait or stature. Routine blood examination was also found normal. 
Surgical excision of the lesion was done under local anesthesia. The excised lesion was transported in $10 \%$ formalin to the laboratory for histopathological examination. Periodontal dressing was placed on the operated area, and the patient was given post-operative instructions; after one week, the dressing was removed. Lesion was completely healed after one month follow-up. On gross examination, the tissue was rubbery soft, well circumscribed, and non-infiltrating.
Microscopic examination revealed sheets of mature adipocytes containing clear cytoplasm and eccentric nucleus, with no evidence of cellular atypia or metaplasia (Figure 2).The whole lesion was surrounded by atrophic, parakeratinised stratified squamous epithelium (Figure 3). Based on the histopathological features, the diagnosis of lipoma was made.

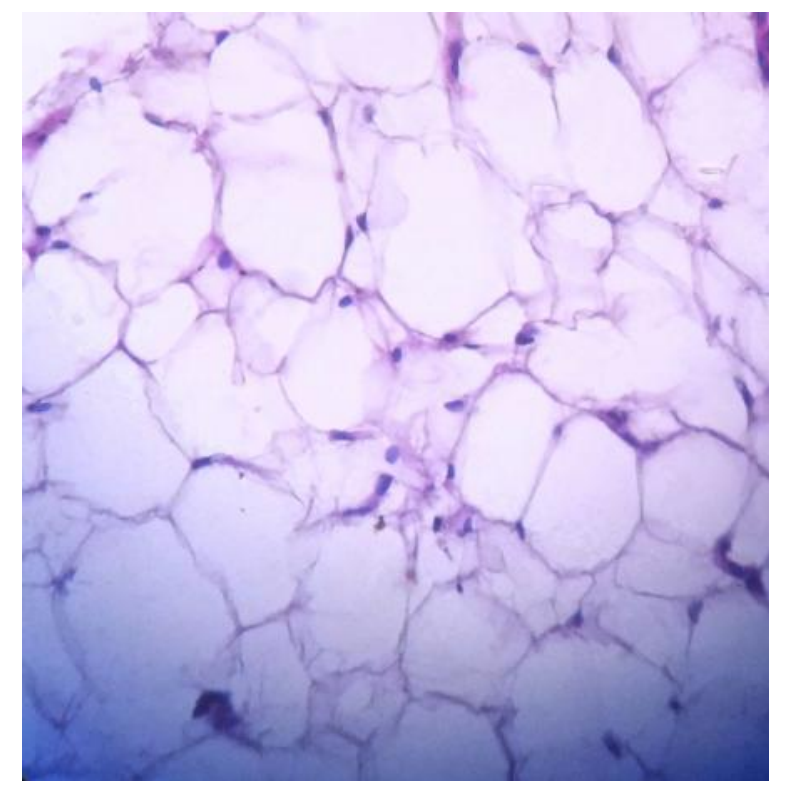

Figure 2: $\mathrm{H} \& \mathrm{E}$ stain $40 \mathrm{X}$ view showing adipocytes with peripherally placed nucleus.

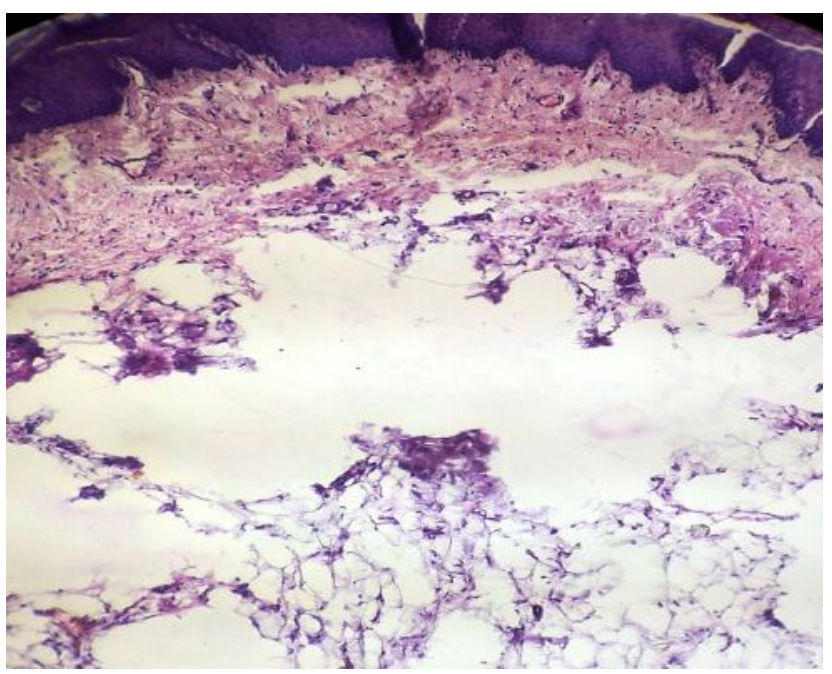

Figure 3: $\mathrm{H}$ \& E stain $100 \mathrm{X}$ view showing stratified squamous epithelium with adipocytes and fibrous connective tissue. 


\section{Discussion}

The lipoma is benign tumor of the adipose tissue but its presence in the oral and oropharyngeal region is relatively uncommon. Lipids unavailable for metabolism coupled with the autonomous growth of a lipoma have rendered it to be a true benign neoplasm.

Oral lipomas are slow growing masses, and patients commonly present with a wellcircumscribed nodule that has been developing for several years. Generally, their prevalence does not differ with gender, although a predilection for men has been reported, and they occur most often in patients older than 40 years. Clinically, oral lipomas generally present as mobile, painless submucosal nodules, with yellowish tinge. Because of these clinical features, other lesions, such as oral dermoid and epidermoid cysts and oral lymphoepithelial cysts must be considered in the differential diagnosis of oral lipomas. Oral dermoid and epidermoid cysts also present as submucosal nodules however, typically, occur on the midline of the floor of the mouth. Most oral lymphoepithelial cysts are found on the floor of the mouth, soft palate and mucosa of the pharyngeal tonsil, which are uncommon sites for oral lipomas. These cysts also differ from oral lipomas in that the nodules are usually small at the time of diagnosis and usually occur in the first to third decade of life. Because an oral lipoma can occasionally present as a deep nodule with normal surface colour, salivary gland tumors and benign mesenchymal neoplasms should also be included in the differential diagnosis. ${ }^{1}$

Lipomas are not very different in microscopic appearance from the surrounding fat. Like fat, they are composed of mature fat cells, but the cells vary slightly in size and shape and are somewhat larger, measuring up to $200 \mathrm{~mm}$ in diameter Lipomas are occasionally altered by the admixture of other mesenchymal elements that comprise an intrinsic part of the tumor. The most common element is fibrous connective tissue, which is often hyalinized and may or may not be associated with the capsule or the fibrous septa. Lipomas with these features are often classified as fibrolipomas. ${ }^{1}$ Fibrolipoma differs from the classic variant because mature adipose tissue is interspersed by bands of connective tissue. ${ }^{6}$ Quite often, however, lesional fat cells are seen as "infiltrate" into surrounding tissues, perhaps producing long thin extensions of fatty tissue radiating from the central tumor mass. When located within striated muscle, this infiltrating variant is called intramuscular lipoma. Occasional lesions exhibit excess numbers of small vascular channels (angiolipoma), a myxoid background stroma (myxoid lipoma), or areas with uniform spindle shaped cells interspersed among normal adipocytes (spindle cell lipoma). ${ }^{1}$ Pleomorphic lipomas are characterized by presence of spindle cells and bizarre hyperchromatic giant cells. ${ }^{3}$ Most of these microscopic variations do not affect the prognosis, which is usually good. The treatment of oral lipomas, including all the histological variants is simple surgical excision. No recurrence has been observed. Although the growth of oral lipomas is usually limited, they can reach great dimensions, interfering with speech and mastication and reinforcing the need for excision. ${ }^{1}$

In the case presented here, the patient was recalled at regular intervals and no sign of recurrence was reported till six months follow-up.

\section{Conclusion}

Intra oral lipoma is a benign tumor with low prevalence in the oral cavity. Clinicians must be able to recognize the differential diagnosis and correct treatment, surgical excision, to ensure the complete eradication of the tumor.

\section{Financial Support and Sponsorship: Nil}

Conflicts of Interests: There are no conflicts of interests

\section{References}

1. Kaur RP, Kler S, Bhullar A. Intraoral Lipoma: report of 3 cases. Dent Res J (Isfahan) 2011 Winter; 8(1): 48-51.

2. Sekar B, Augustine D, Murali S. Lipoma, a rare intraoral tumor-a case report with review of literature. OMPJ 2011; 2(2): 174-7. 
3. Ranginwala A, Kale H, Modi T, Dave K. Intra-oral lipoma. J Int Clin Dent Res Organ 2010; 2:157-60.

4. Studart-Soares EC, Costa FWG, Sousa FB, Alves APNN, Osterne RLV. Oral lipomas in a Brazilian population: A 10-year study and analysis of 450 cases reported in literature. Med Oral Patol Oral Cir Bucal 2010 Sep 1; 15(5): e691-6.

5. Gulia JS, Yadav SPS, Rana P, Singh S, Duhan A, Sen R, Hooda A. Lipoma Oral Cavity: A Case report with review of literature. IJO 2012; 14(1).

6. Manjunatha BS, Pateel GS, Shah V. Oral Fibrolipoma-A Rare Histological Entity: Report of 3 Cases and Review of Literature. J Dent (Tehran). 2010 Fall; 7(4):226-31. Epub 2010 Sep 30.

\begin{tabular}{|c|l|}
\hline \multicolumn{2}{|c|}{ Access this Article in Online } \\
\hline Q & Website: \\
\hline & www.ijcrims.com \\
& Subject: \\
Quick Response Code & \\
\hline
\end{tabular}

How to cite this article:

Chhaya Mangla, Sukhpal Kaur, Riponjot Singh, Harpreet Kaur Dhanju. (2017). A Case report of Oral lipoma on gingiva - A rare histological entity. Int. J. Curr. Res. Med. Sci. 3(6): 75-79.

DOI: http://dx.doi.org/10.22192/ijcrms.2017.03.06.010 